Dialectologie : corpus, atlas, analyses

\begin{abstract}
Alain CHEVRIER - Le Décasyllabe à césure médiane. Histoire du taratantara. Paris : Classiques Garnier, coll. «Etudes romantiques et dix-neuviémistes » 18, 2011, 405 pages, $49 €$.
\end{abstract}

\title{
Gérald Purnelle
}

\section{(2) OpenEdition}

Journals

Electronic version

URL: http://journals.openedition.org/corpus/2416

DOI: $10.4000 /$ corpus. 2416

ISSN: $1765-3126$

\section{Publisher}

Bases; corpus et langage - UMR 6039

\section{Printed version}

Date of publication: 1 January 2013

Number of pages: 331-333

ISSN: 1638-9808

\section{Electronic reference}

Gérald Purnelle, «Alain cheVRier - Le Décasyllabe à césure médiane. Histoire du taratantara. Paris Classiques Garnier, coll. «Etudes romantiques et dix-neuviémistes » 18, 2011, 405 pages, 49 €. », Corpus [Online], 12 | 2013, Online since 14 May 2014, connection on 23 September 2020. URL : http:// journals.openedition.org/corpus/2416; DOI : https://doi.org/10.4000/corpus.2416 
Alain CHEVRIER - Le Décasyllabe à césure médiane. Histoire du taratantara. Paris : Classiques Garnier, coll. «Etudes romantiques et dix-neuviémistes »18, 2011, 405 pages, $49 €$.

L'ouvrage d'Alain Chevrier relève de ce qu'il nomme lui-même la «morphologie poétique », c'est-à-dire l'étude des formes poétiques, qui contribue à l'histoire littéraire. Il y trace l'histoire détaillée d'un vers particulier, le décasyllabe à césure médiane (en 5-5). Ce vers est traditionnellement baptisé taratantara, en une onomatopée qui calque la longueur syllabique de ses hémistiches.

L'intérêt de l'ouvrage réside à la fois dans sa structure linéaire (il s'agit d'une " histoire » de ce vers) et dans ses approches multiples.

Notons d'emblée que, pour chaque époque, les poètes ayant pratiqué ce vers sont évoqués l'un après l'autre, et que leur usage du vers est illustré par au moins un poème, souvent cité en entier. L'ouvrage est donc aussi, et très plaisamment, une anthologie historique du décasyllabe à césure médiane, qui permet au lecteur curieux de (re)découvrir des noms et des textes peu connus, voire inconnus. Ce seul aspect rend donc déjà l'ouvrage fort utile et agréable.

Mais histoire et analyse structurent l'ouvrage dans sa dimension scientifique. Fondé sur « de nombreux documents peu connus, méconnus ou inconnus ", l'ouvrage commence par l'exploration de la préhistoire du vers et par une esquisse sommaire des «Débuts du décasyllabe », qui paraît plonger ses racines dans des modèles latins médiévaux.

L'auteur relève les différentes structures attestées pour les vers de dix syllabes (4-6 et 5-5, mais aussi 6-4), mais, sur un plan analytique et méthodologique, on regrettera qu'à ce stade soit posé sans discussion l'objet « décasyllabe », supposé subsumer ces trois schémas ou « vers » morphologiquement différents, sans autre forme de questionnement. Deux schémas aussi différents que 4-6 et 5-5 forment-ils, métriquement parlant, un même vers? La réponse affleure plusieurs fois dans le corps de l'ouvrage, dans les cas où (chez tel poète, dans tel poème), ils se mêlent (quand des 5-5 apparaissent en contexte de 6-6), mais elle n'est guère examinée pour elle-même. 


\section{Comptes rendus}

Plus longue et intéressante est l'étude de l'origine et du sens du terme étrange qui sert à nommer le 5-5 (décasyllabe à césure médiane), taratantara, de sa première apparition dans un vers du poète latin Ennius, où il désigne le son de la trompette, jusqu'à sa mention dans les traités de versification des XIX et $\mathrm{XX}^{\mathrm{e}}$ siècles, en passant surtout par ses premières utilisations, pour désigner le vers, par Bonaventure des Périers ( $\mathrm{XVI}^{\mathrm{e}} \mathrm{s}$.) et Régnier-Desmarais (XVII ${ }^{\mathrm{e}} \mathrm{s}$.).

Le parcours historique commence par un court chapitre consacré au Moyen Age, où domine l'étude de La Cantilène de sainte Eulalie et des chansons médiévales où le vers apparaît. La suite distingue deux moments particuliers et plus importants de l'histoire: la Renaissance et l'âge baroque, d'une part, le $\mathrm{XIX}^{\mathrm{e}}$ siècle d'autre part.

Pour le premier de ces deux chapitres, un traitement particulier est consacré à un poème de 1367 vers, inséré dans un ouvrage peu connu, paru dans la première moitié du XVI siècle : Le Jardin amoureux de Christophe de Barrouso. La longueur des vers n'y est pas fixe et syllabique, mais variable. Le poème contient néanmoins de nombreux décasyllabes, dont des 5-5. En une longue analyse de trente pages, Alain Chevrier conclut, pour expliquer cette versification complexe, à une adaptation française de l'arte mayor espagnol.

L'étude de la place du taratantara dans la métrique des vers mesurés de Baïf, issus, notamment, de l'adaptation en français de la strophe saphique, et où apparaissent des 5-5 parmi des 4-6, est également éclairante, tout comme celle de la résurgence ou réinvention du décasyllabe en 5-5 au XIX ${ }^{\mathrm{e}}$ siècle, attribuable à Marceline Desbordes-Valmore, avant que le Parnasse en fasse un nouvel usage.

Après un chapitre consacré au $\mathrm{Xx}^{\mathrm{e}}$ siècle, une brève «récapitulation» esquisse une rapide histoire du décasyllabe (tous schémas confondus) puis balise les quelques moments et observations d'une histoire riche, parsemée de creux et de temps forts, de discontinuité et de reprises.

On doit reformuler le regret exprimé au début de ce compte rendu, pour ce qui touche les conclusions qu'il eût été possible de tirer de cette histoire. La question de la concurrence 


\section{Comptes rendus}

des différents schémas, 4-6, 6-4 et 5-5, n'est guère abordée, et nullement de façon systématique et synthétique. Or on observe qu'à la Renaissance, des poèmes mêlent ces schémas, notamment les poèmes transposant en français la strophe saphique à base d'hendécasyllabes. A nouveau, sans qu'il y ait de lien, le phénomène réapparait chez certains poètes à la fin du XIX ${ }^{\mathrm{e}}$ siècle (Rimbaud). Entre ces deux moments de l'histoire a pesé un interdit à la fois théorique et pratique, excluant, pour le poète, le droit de mêler des schémas pairs (4-6, 6-4) avec un schéma à base impaire (5-5). Cet interdit n'est guère étudié dans l'ouvrage.

Il y a, théoriquement, plusieurs situations possibles :

a) des poèmes à schéma unique, obéissant à l'interdit du mélange, ou produits à un moment où ce mélange est tout bonnement impensable ; soit des poèmes entièrement en 4-6 et d'autres entièrement en 5-5;

b) des poèmes où le mélange est jugé possible et pratiqué ; par exemple (sans doute le plus fréquent), un poème où dominent les 4-6, mais où le schéma 5-5 apparaît minoritairement (parfois une seule occurrence);

c) des poèmes où les schémas à base paire et impaire sont mêlés sans qu'une dominante soit perceptible.

Il eût été utile et éclairant de dresser une chronologie de ces trois types théoriques, d'évaluer diachroniquement leur présence ou leur absence à chaque époque, et de distinguer clairement, récapitulativement et pour chaque période, les poètes « réguliers » (dont les poèmes sont en 5-5) et ceux qui consentent à mêler schémas pairs et impairs. Le tout en mobilisant des notions telles que norme, interdit et transgression. Répétons-le, l'enjeu est théorique : il porte sur la question de savoir si, pour une époque ou pour un poète, le 4-6 et le 5-5 sont deux vers différents ou une même mètre; si le nombre (10) prime sur la structure ; s'il existe $u n$ décasyllabe ou plusieurs.

Quoi qu'il en soit de cette réserve, le matériau fourni par cet ouvrage, mais aussi les divers éclairages analytiques ou théoriques qui le parsèment, en font une source précieuse pour l'étude du vers (en général) et pour la poursuite de la réflexion théorique.

Gérald PURNELLE

LASLA, Université de Liège 